\title{
Alzheimer's disease severity and its association with patient and caregiver quality of life in Japan: results of a community-based survey
}

William Montgomery ${ }^{1}$, Amir Goren ${ }^{2}$, Kristin Kahle-Wrobleski ${ }^{3}$, Tomomi Nakamura ${ }^{4}$ and Kaname Ueda ${ }^{5^{*}}$

\begin{abstract}
Background: Alzheimer's disease (AD) dementia, a progressive neurodegenerative disease, exerts significant burden upon patients, caregivers, and healthcare systems globally. The current study investigated the associations between $A D$ dementia patient disease severity and health-related quality of life (HRQoL) of both patients (proxy report) and their caregivers living in Japan, as well as caregiving-related comorbidities such as depression.

Methods: This cross-sectional study used self-reported data from caregivers of people diagnosed with AD dementia by a healthcare provider in Japan. Caregivers were identified via online panels and invited to participate in an online survey between 2014 and 2015. Caregivers completed survey items for themselves, in addition to providing proxy measures for patients with AD dementia for whom they were caring. Patient and caregiver HRQoL was measured using the EuroQoL 5-Dimension (EQ-5D). Additional outcomes for caregivers of AD dementia patients included the Patient Health Questionnaire (PHQ-9) of depressive symptomology, as well as comorbidities experienced since initiating caregiving for their AD dementia patients. These outcomes were examined as a function of AD dementia severity, as measured by long-term care insurance (LTCI) categories. Bivariate analyses between $\mathrm{LTCl}$ and outcomes were conducted using independent t-tests and chi-square tests. Multivariable analyses, controlling for potential confounders, were conducted using generalized linear models (GLMs) specifying a normal distribution.
\end{abstract}

Results: Across 300 caregiver respondents, multivariable results revealed that increasing AD dementia severity was significantly associated with poorer patient and caregiver EQ-5D scores and a high proportion of caregivers (30.0\%) reported PHQ-9 scores indicative of major depressive disorder (MDD). The most frequent comorbidities experienced after becoming caregivers of AD dementia patients included hypertension (12.7\%) and insomnia (11.0\%).

Depression and other comorbidities did not differ significantly by patient severity.

Conclusions: The current study provides unique insight into the specific degree of incremental burden associated with increasing AD dementia severity among patients and caregivers alike. Importantly, greater disease severity was associated with poorer quality of life among both patients and caregivers. These results suggest that earlier detection and treatment of AD dementia may provide an opportunity to reduce the burden of disease for patients, caregivers, and society at large.

Keywords: Alzheimer's disease dementia, Caregiving burden, Disease severity, Quality of life, Depression, Japan

\footnotetext{
* Correspondence: ueda_kaname@lilly.com

${ }^{5}$ Health Outcomes, Health Technology Assessment, \& Real World Evidence,

Medical Development Unit, Eli Lilly Japan K.K, 5-1-28, Isogami-dori, chuou-ku,

Kobe 651-0086, Japan

Full list of author information is available at the end of the article
}

(c) The Author(s). 2018 Open Access This article is distributed under the terms of the Creative Commons Attribution 4.0 International License (http://creativecommons.org/licenses/by/4.0/), which permits unrestricted use, distribution, and reproduction in any medium, provided you give appropriate credit to the original author(s) and the source, provide a link to the Creative Commons license, and indicate if changes were made. The Creative Commons Public Domain Dedication waiver (http://creativecommons.org/publicdomain/zero/1.0/) applies to the data made available in this article, unless otherwise stated. 


\section{Background}

Alzheimer's disease (AD) dementia is a progressive neurodegenerative disease that poses a significant burden to patients, caregivers, healthcare systems, and society. Disease symptomatology includes impaired cognition (e.g., memory difficulties) and is thus associated with difficulty in performing daily activities and consequent functional dependence on others for help. These symptoms and the progressive nature of $\mathrm{AD}$ dementia can result in increasing degrees of care required from formal (institutional$\mathrm{ized} /$ paid) and informal (family) caregivers [1]. The cause of AD dementia has not been fully identified and there is currently no cure; available treatments target cognitive symptoms and the management of behavioral symptoms [2].

Critically, the health, societal, and economic burden associated with $\mathrm{AD}$ dementia may increase as the severity of the disease increases [3-5], with both formal and informal $\mathrm{AD}$ dementia care contributing to this societal burden [6]. Disease severity has been found to be an independent predictor of time to institutionalized care and increased costs [7], while further studies have reported that increasing disease severity, including behavioral disturbances and greater symptomatology, is predictive of higher care costs [8-11]. A European/US based review reported that costs were more than doubled when comparing patients with severe dementia with those with mild dementia [12].

The high cost of informal care in AD dementia highlights the unique burden and responsibility placed on families of persons with AD dementia. Many AD dementia patients depend on care from family members due to the associated impairments in functioning and cognition that are hallmarks of this progressive disease. Notably, the care needs of patients with $\mathrm{AD}$ dementia can increase significantly as disease severity progresses [13], and studies have shown that family caregivers have a greater risk of medical illness, psychological impairment, disruption of personal and professional roles, and critically, an increased risk of mortality [3, 14-16].

Globally, the impact on caregivers has shown a range of negative outcomes in the context of $\mathrm{AD}$ dementia care $[17,18]$, including physical $[15,19]$, psychological [20, 21], social [22], and financial [23] consequences, with similar findings beginning to emerge in developing countries [18]. These findings have been documented in Japan, with research suggesting that family caregivers of elderly patients with dementia can experience significant burden and face various challenges associated with providing dementia-related care [24-31]. In a precursor to the present study, caregivers of individuals with $\mathrm{AD}$ dementia or other types of dementia in Japan were compared with matched non-caregivers in terms of their characteristics and health outcomes [32]. Consistent with international research, caregivers experienced significant impairments relative to their non-caregiver counterparts. Caregivers vs. non-caregivers reported poorer quality of life, lower levels of work productivity, and higher rates of mental health issues and healthcare utilization. Whereas $\mathrm{AD}$ dementia caregivers represent a vulnerable population at risk of negative health outcomes (especially as the disease progresses), evidence also suggests that some caregivers experience positive effects associated with caring for a family member diagnosed with a chronic or terminal disease, most often in the form of benefit-finding (e.g., strengthening of familial ties) or enhanced meaning [33-36].

Japan is ranked among the countries with the highest prevalence, with a 2010 study estimating 2.5 million adults suffering from dementia, a number predicted to reach 7.3 million by 2025 [37, 38]. To address the needs of care among a growing aging population, Japan implemented an insurance program and long-term care approach in 2000 that focused on consistent evaluation of the elderly and increased care and financial support for family caregivers [39]. AD dementia patients are majority users of long-term care insurance (LTCI) support for their care services. Despite this, there is notably little population-level empirical data concerning the specific impact of increasing disease severity on patient and caregiver outcomes, particularly assessing the relationship between these factors in the same study population. The current study provides important and novel insights into the incremental burden associated with increasing patient disease severity, and the relationship between burden and both patients' and caregivers' health outcomes. This further detailed quantification of burden, including among those with mild disease, can help provide valuable insight into the experience of patients and informal caregivers, guide decisions concerning burden and care needs, and help quantify the potential benefits/cost savings of effective management of this disease. The authors hypothesized that increasing patient disease severity would be associated with poorer patient and caregiver outcomes in Japan.

\section{Methods \\ Sample}

This cross-sectional study collected new data from caregivers of people with a clinical diagnosis of AD dementia who were previously identified in the 2012/2013 Japan National Health and Wellness Surveys (NHWS; internet-based questionnaires sampling adults aged 18 and older), with additional caregivers supplemented from Lightspeed Research (LSR) opt-in ailment panels. Convenience sampling, stratified by sex and age, was implemented to achieve demographic characteristics that 
match the adult population in Japan. Further details about the NHWS and respondent recruitment are provided in Laks et al. [40] and Goren et al. [32]. Inclusion criteria were: adults (18 years of age or older); caring for an individual diagnosed with AD dementia (per caregiver self-report); not receiving payment as a form of employment for caregiving duties; and providing informed consent. These new survey data were collected between December 2014 to January 2015. A sample of 300 caregivers was included in this analysis, including 119 current caregivers who were successfully recruited from the 2012 and 2013 NHWS and an additional 181 current caregivers who were recruited from the separate ailment panel source. Caregivers provided survey responses for themselves in addition to proxy measures on behalf of the AD dementia patient for whom they provided care.

\section{Measures \\ Sociodemographics and health characteristics}

Sociodemographic variables included sex and age for both patients and caregivers. An additional background variable for patients included LTCI levels reported by their caregivers. The implementation of LTCI in Japan required detailed assessments of patients' needs to effectively assign care resources. The Government-certified Disability Index (GCDI) is an 85-item measure developed to serve this purpose [41], providing a score from 0 to 5 that dictates the amount spent on services for a patient in each category and enables the severity of patient disease to be inferred. LTCI levels in the current study were trichotomized for analysis into categories reflecting increasing severity mapping roughly onto the GCDI levels: (1) low = no long-term nursing care insurance, support levels 1 or 2 , or level not known; (2) medium $=$ nursing care levels 1,2 , or 3 ; and (3) high = nursing care levels 4 or 5 .

Additional background variables for caregivers included marital status, number of children in the household, change in employment status due to caregiving for the $\mathrm{AD}$ dementia patient, relationship with patient, and level of involvement with caregiving.

\section{Patient disease severity}

Patient disease severity was reported by caregivers based on patients' LTCI scores. Previous research has demonstrated that LTCI is associated with other measures of disease severity [42], supporting its use as a proxy measure of $\mathrm{AD}$ dementia severity. Other measures that are associated with LTCI scores include the Short-Memory Questionnaire (SMQ) $[43,44]$ and an index of patient dependence.

\section{Comorbidities}

Charlson comorbidity index (CCI) [45] scores sum the weighted presence of comorbid conditions contributing to mortality risk. Higher scores indicate greater comorbid burden for the caregiver. Quan et al.'s [46] CCI scoring was implemented, as this updates the original algorithm on the basis of a more recent replication, with updated weighting and fewer conditions. Caregivers were asked if they had experienced these comorbidities since becoming caregivers of the $\mathrm{AD}$ dementia patients.

\section{Health related quality of life}

Two forms of the EuroQoL-5Dimension (EQ-5D) with 3 levels (Japanese version and value set), a widely used measure of health status, were used: one to assess the caregiver's HRQoL, and a proxy version for the caregiver to rate the patient's HRQoL from the patient's perspective $[47,48]$. The EQ-5D encompasses five dimensions (i.e., mobility, self-care, usual activities, pain/discomfort, and anxiety/depression), with responses corresponding to 3 levels of severity. The EQ-5D also generates a single health status index utility value ranging from 0 (death) to 1 (perfect health) $[49,50]$.

\section{Depression}

The Patient Health Questionnaire (PHQ)-9 [51, 52] was used to assess the frequency of caregivers' depressive symptoms. A total score of 10 or higher [53] was used as an indicator of major depressive disorder (MDD). A "diagnosed depression" question was also included among other comorbidities experienced since initiating caregiving.

\section{Analysis}

Descriptive analyses were conducted on all study variables of interest; these included $n \mathrm{~s}$ and \%s for categorical variables and means, medians, standard deviations (SDs), and minimum and maximum values for continuous variables. Bivariate associations were examined between the primary independent variable, LTCI, and several HRQoL and comorbidity dependent measures using chi-square tests for categorical variables (e.g., PHQ-9 MDD cut-off) and $\mathrm{t}$-tests for continuous variables (i.e., EQ-5D), with two-tailed $p$-values indicating statistical significance. Results of these bivariate analyses helped inform variables that were included as covariates in multivariable analyses. Covariates were identified according to statistically significant differences in bivariate comparisons $(p<.05)$ across the AD dementia severity categories. Some core variables of conceptual interest (e.g., age and gender) were included regardless of their association with the severity categories.

Multivariable analyses were used to assess patient and caregiver outcomes as a function of patient severity (i.e., burden as a function of LTCI levels, adjusting for potential 
measured confounders). The final list of identified covariates included: caregiver gender, caregiver age, caregiver employment status, caregiver marital status, percentage average daily care for which caregiver is responsible, percentage average daily care from visiting nurse/home-helper, total average hours of care required per day, years providing care for AD dementia patient, relationship between caregiver and patient, patient age, and patient gender. Separate generalized linear models (GLMs) specifying appropriate distributions (e.g., identity link function for normally distributed continuous outcomes, such as HRQoL) were chosen for each outcome of interest that was associated significantly with AD dementia severity in bivariate analyses. Multivariable models included exploratory interaction terms between age categories and employment status of caregivers on both caregiver and patient outcomes. These interaction terms were included to test whether the relationship between employment status and outcomes depended upon age of caregivers. These interaction terms were expected to account for cultural expectations surrounding age and work status (e.g., unemployment among younger adults may be associated with greater reductions in quality of life relative to older adults).

\section{Results}

\section{Patient demographics}

Across 299 patients (excluding 1 outlier with a caregiver-reported patient age of 4 years), the mean age was 83.7 years $(S D=7.6$, median $=84)$, with minimum and maximum values of 38 and 106 years, and 78.7\% of patients were female.

LTCI was used as a surrogate patient disease severity measure, with $12.3 \%$ of caregivers $(n=37$ in this "low severity" group) indicating that they did not know their patients' LTCI $(n=3)$ or that their patients had no LTCI $(n=15)$ or support level $1(n=7)$ or $2(n=12)$; $63.7 \%$ $(n=191$ in this "medium severity" group) indicating that patients had nursing care level $1(n=52), 2(n=75)$, or $3(n=64)$; and $24.0 \%(n=72$ in this "high severity" group) indicating that patients had nursing care level $4(n=43)$ or $5(n=29)$ (see Table 1$)$.

\section{Caregiver demographics}

Across 300 caregivers (see Table 2), the average caregiver age was 53.9 years $(S D=11.0$, median $=55)$, with minimum and maximum values of 20 and 96 years. A total of $61.0 \%$ were between the ages of 50 and 64 and $10.7 \%$ were 65 years of age or older. Of the caregiver sample, $55.0 \%$ were male, $62.3 \%$ were married or living with a partner, $51.0 \%$ had at least one dependent adult in the household, $17.7 \%$ had at least one dependent child in
Table 1 Demographics and characteristics of patients (as reported by caregivers)

\begin{tabular}{|c|c|c|c|}
\hline \multirow[t]{2}{*}{ Characteristic } & & \multicolumn{2}{|l|}{ Total } \\
\hline & & $\%$ & $n$ \\
\hline \multicolumn{2}{|c|}{ Patient age (mean (SD), min to max) } & $83.70(7.63)$ & 38 to 106 \\
\hline \multirow[t]{4}{*}{ Age category } & $<80$ & $23.7 \%$ & 71 \\
\hline & $80-84$ & $30.3 \%$ & 91 \\
\hline & $85-89$ & $23.7 \%$ & 71 \\
\hline & $90+$ & $22.3 \%$ & 67 \\
\hline \multirow[t]{2}{*}{ Gender of patient } & Female & $78.7 \%$ & 236 \\
\hline & Male & $21.3 \%$ & 64 \\
\hline \multirow[t]{9}{*}{ Level of LTCI certification } & No LTCI & $5.0 \%$ & 15 \\
\hline & Support level 1 & $2.3 \%$ & 7 \\
\hline & Support level 2 & $4.0 \%$ & 12 \\
\hline & $\begin{array}{l}\text { Nursing care } \\
\text { level } 1\end{array}$ & $17.3 \%$ & 52 \\
\hline & $\begin{array}{l}\text { Nursing care } \\
\text { level } 2\end{array}$ & $25.0 \%$ & 75 \\
\hline & $\begin{array}{l}\text { Nursing care } \\
\text { level } 3\end{array}$ & $21.3 \%$ & 64 \\
\hline & $\begin{array}{l}\text { Nursing care } \\
\text { level } 4\end{array}$ & $14.3 \%$ & 43 \\
\hline & $\begin{array}{l}\text { Nursing care } \\
\text { level } 5\end{array}$ & $9.7 \%$ & 29 \\
\hline & Don't know & $1.0 \%$ & 3 \\
\hline \multirow[t]{3}{*}{ Category of LTCI certification } & Low severity & $12.3 \%$ & 37 \\
\hline & Medium severity & $63.7 \%$ & 191 \\
\hline & High Severity & $24.0 \%$ & 72 \\
\hline
\end{tabular}

Note. $L T C l$ long-term nursing care insurance, $S D$ standard deviation

the household, and $26.7 \%$ reported changing employment status due to caregiving.

Among those who recalled the total duration of caregiving $(n=276), 55.8 \%$ had provided at least 4 years of care; $13.0 \%$ had provided at least 10 years of care. Among all caregivers, $39.7 \%$ were secondary caregivers, $30.0 \%$ were primary caregivers but shared responsibilities, and 30.3\% were the only primary caregivers. Caregivers were personally responsible for an average of $13.0 \mathrm{~h}(S D=8.01)$ of care per day, with $48.0 \%$ of respondents responsible for $13 \mathrm{~h}$ of care per day or greater. Data were collected regarding the caregiver's relationship to the patient (e.g., parent, spouse, offspring). Age and relationship data revealed that caregivers responded inconsistently regarding the direction of the parent vs. offspring relationship; therefore, responses were recoded into more logically defensible categories: offspring of patient (i.e., caregiver was at least 20 years younger than the patient), spouse/sibling/parent of patient (i.e., caregiver was older or no more than 20 years younger than the patient), or in-law/extended family/not related to patient (i.e., caregiver explicitly selected one of these options). 
Table 2 Demographics and characteristics of AD dementia caregivers

\begin{tabular}{|c|c|c|c|}
\hline \multirow[t]{2}{*}{ Characteristic } & & \multicolumn{2}{|l|}{ Total } \\
\hline & & $\%$ & $n$ \\
\hline \multicolumn{2}{|l|}{ Caregiver age (mean (SD), min to max) } & $53.89(11.02)$ & 20 to 96 \\
\hline \multirow[t]{5}{*}{ Age category } & $18-29$ & $3.0 \%$ & 9 \\
\hline & $30-39$ & $7.7 \%$ & 23 \\
\hline & $40-49$ & $17.7 \%$ & 53 \\
\hline & $50-64$ & $61.0 \%$ & 183 \\
\hline & $65+$ & $10.7 \%$ & 32 \\
\hline \multirow[t]{2}{*}{ Gender of caregiver } & Female & $45.0 \%$ & 135 \\
\hline & Male & $55.0 \%$ & 165 \\
\hline \multirow[t]{3}{*}{ Marital status } & Single & $28.3 \%$ & 85 \\
\hline & Married / living with partner & $62.3 \%$ & 187 \\
\hline & Divorced / separated / widowed & $9.3 \%$ & 28 \\
\hline \multirow[t]{5}{*}{ Adults (18+) dependent in household } & 0 & $29.0 \%$ & 87 \\
\hline & 1 & $20.0 \%$ & 60 \\
\hline & 2 & $20.7 \%$ & 62 \\
\hline & 3 & $15.0 \%$ & 45 \\
\hline & $4+$ & $15.3 \%$ & 46 \\
\hline \multirow[t]{3}{*}{ Children $(<18)$ dependent in household } & 0 & $82.3 \%$ & 247 \\
\hline & 1 & $8.7 \%$ & 26 \\
\hline & $2+$ & $9.0 \%$ & 27 \\
\hline \multirow[t]{2}{*}{ Employed } & $\begin{array}{l}\text { Not employed, disabled, retired, student, } \\
\text { or homemaker }\end{array}$ & $31.0 \%$ & 93 \\
\hline & Full-time, part-time, or self-employed & $69.0 \%$ & 207 \\
\hline \multirow{2}{*}{$\begin{array}{l}\text { Change in employment status due to caregiving for } \\
\text { AD dementia patient }\end{array}$} & No & $73.3 \%$ & 220 \\
\hline & Yes & $26.7 \%$ & 80 \\
\hline \multirow[t]{6}{*}{ Years providing care for the AD dementia patient } & $<2$ years & $7.0 \%$ & 21 \\
\hline & 2 to $<4$ years & $33.7 \%$ & 101 \\
\hline & 4 to $<6$ years & $22.0 \%$ & 66 \\
\hline & 6 to $<10$ years & $17.3 \%$ & 52 \\
\hline & $10+$ years & $12.0 \%$ & 36 \\
\hline & Can't recall & $8.0 \%$ & 24 \\
\hline \multirow[t]{3}{*}{ Caregiving role for $\mathrm{AD}$ dementia patient } & Primary and only caregiver & $30.3 \%$ & 91 \\
\hline & $\begin{array}{l}\text { Primary and sharing responsibilities with } \\
\text { another person }\end{array}$ & $30.0 \%$ & 90 \\
\hline & Secondary caregiver & $39.7 \%$ & 119 \\
\hline \multirow[t]{5}{*}{ Hours/week 'personally responsible for providing care' } & $1-4 \mathrm{~h}$ & $21.7 \%$ & 65 \\
\hline & $5-12 \mathrm{~h}$ & $16.3 \%$ & 49 \\
\hline & $13-24 h$ & $26.0 \%$ & 78 \\
\hline & 25-72 h ( $1-3$ days) & $20.0 \%$ & 60 \\
\hline & 73-168 h (3-7 days) & $16.0 \%$ & 48 \\
\hline
\end{tabular}

Note. $A D$ Alzheimer's disease, $S D$ standard deviation

HRQoL for caregivers and patients

EQ-5D patient proxy index values ranged from -0.110 to 1.000 , with a mean of $0.542(S D=0.224)$ and median of 0.595 . EQ-5D caregiver index values ranged from -0.110 to 1.000 , with a mean score of $0.809(\mathrm{SD}=0.216)$ and median of 0.785 (see Fig. 1).

The most common comorbidities that caregivers experienced since becoming caregivers included insomnia 


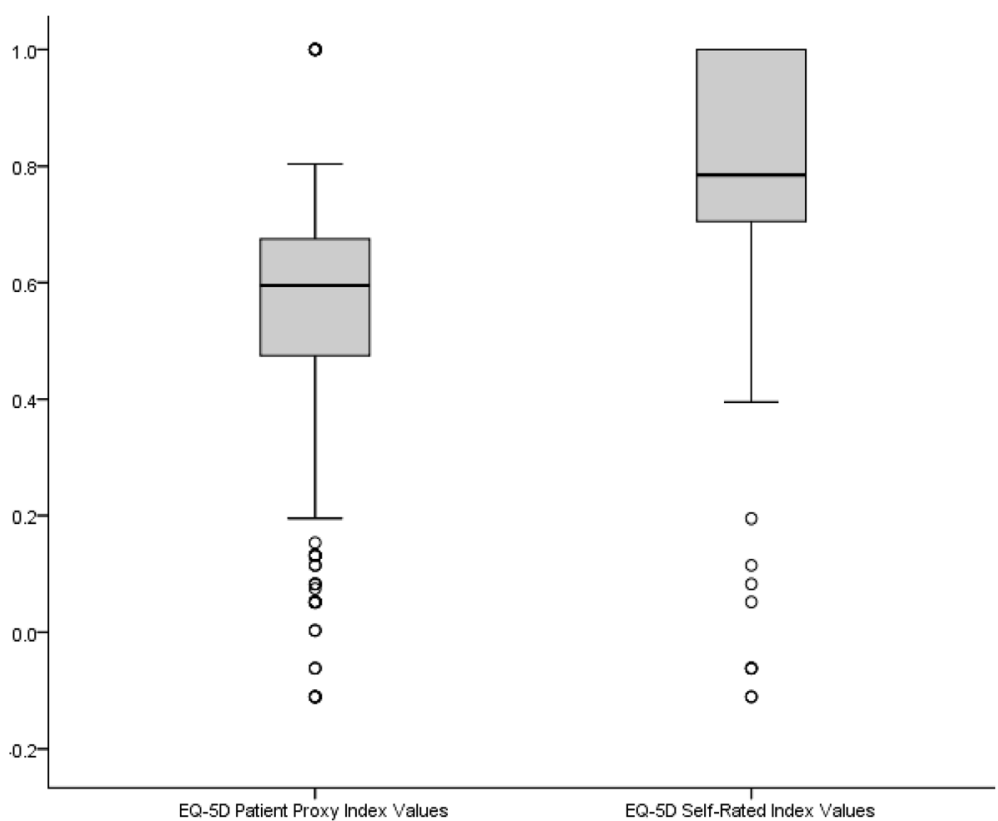

Fig. 1 Box and whisker plot for EQ-5D patient proxy and caregiver values. Note: The ends of the boxes shown above depict the lower and upper quartiles while the horizontal line in the middle of each box depicts the median value. The whiskers extending from each box show the range of the lowest and highest observation. Outliers are identified with small circles (1.5× Interquartile Range)

(11.0\%) and hypertension (12.7\%). Most caregivers (92.0\%) reported no diagnosed comorbidities since becoming caregivers, however. A total of 90 re-contact caregivers (30.0\%) experienced MDD as indicated via PHQ-9 scores $\geq 10$ (see Table 3).
Bivariate and multivariable results

Bivariate analyses demonstrated that $\mathrm{AD}$ dementia severity was associated with poorer patient HRQoL as measured by EQ-5D patient proxy scores. AD dementia patients at the low severity LTCI level experienced

Table 3 Comorbidity of caregivers

\begin{tabular}{|c|c|c|c|}
\hline \multirow[t]{2}{*}{ Characteristic } & & \multicolumn{2}{|l|}{ Total } \\
\hline & & $\%$ & $n$ \\
\hline \multirow[t]{5}{*}{ Depression (PHQ-9 scores) } & None $(0-4)$ & $41.7 \%$ & 125 \\
\hline & Mild depression (5-9) & $28.3 \%$ & 85 \\
\hline & Moderate depression (10-14) & $13.0 \%$ & 39 \\
\hline & Moderately severe depression (15-19) & $10.0 \%$ & 30 \\
\hline & Severe depression (20-27) & $7.0 \%$ & 21 \\
\hline \multirow[t]{2}{*}{ MDD (PHQ-9 scores) } & None $(<10)$ & $70.0 \%$ & 210 \\
\hline & MDD (10+) & $30.0 \%$ & 90 \\
\hline \multirow[t]{6}{*}{ Diagnosed comorbidities } & Depression & $4.7 \%$ & 14 \\
\hline & Insomnia & $11.0 \%$ & 33 \\
\hline & Anxiety & $1.7 \%$ & 5 \\
\hline & Hypertension & $12.7 \%$ & 38 \\
\hline & Pain & $5.7 \%$ & 17 \\
\hline & Diabetes (Type1/2) & $1.0 \%$ & 3 \\
\hline \multirow[t]{3}{*}{$\mathrm{CCl}$ experienced since caregiving } & 0 & $92.0 \%$ & 276 \\
\hline & 1 & $2.7 \%$ & 8 \\
\hline & $2+$ & $5.3 \%$ & 16 \\
\hline
\end{tabular}


significantly and substantially higher EQ-5D proxy health utility scores $(M=0.69 S D=0.16)$ than did those AD dementia patients at the medium severity LTCI level $(M=$ $0.60, S D=0.16)$ as well as patients at the high severity LTCI level $(M=0.31, S D=0.24) ; p$ 's $<.05$. AD dementia severity significantly predicted EQ-5D scores for patients after adjusting for covariates in the multivariable model (see Table 4, Fig. 2).

$\mathrm{AD}$ dementia severity was also associated with poorer caregiver quality of life as measured by EQ-5D scores.
Caregivers of $\mathrm{AD}$ dementia patients at the high severity level of LTCI experienced significantly lower EQ-5D health utility scores $(M=0.75, S D=0.28)$ than did caregivers of AD dementia patients at the low severity LTCI level $(M=0.84, S D=0.17), p<.05$. EQ-5D scores did not significantly differ between caregivers of patients at medium severity and low severity LTCI levels, however. After controlling for covariates, AD dementia severity remained a significant predictor of caregiver EQ-5D scores in the multivariable model (see Table 5). In both

Table 4 Results of generalized linear regression model of patient EQ-5D proxy index values as a function of LTCI \& covariates $(n=300)$

\begin{tabular}{|c|c|c|c|c|c|c|}
\hline Characteristic & Reference Category & Variables & B & $\begin{array}{l}95 \% \\
\mathrm{LCL} \\
\end{array}$ & $\begin{array}{l}95 \% \\
\mathrm{UCL} \\
\end{array}$ & $\begin{array}{l}P \text { - } \\
\text { Value }\end{array}$ \\
\hline \multicolumn{3}{|l|}{ (Intercept) } & 0.761 & 0.629 & 0.894 & $\begin{array}{l}< \\
0.001\end{array}$ \\
\hline \multicolumn{7}{|l|}{ Patient characteristics } \\
\hline \multirow[t]{2}{*}{ AD dementia severity } & \multirow[t]{2}{*}{ Low severity } & High severity & -0.340 & -0.414 & -0.267 & $\begin{array}{l}< \\
0.001\end{array}$ \\
\hline & & Medium severity & $-\overline{0}-072$ & -0.134 & -0.01 & 0.022 \\
\hline Sex & Female & Male & -0.030 & -0.078 & 0.018 & 0.220 \\
\hline \multirow[t]{2}{*}{ Age } & \multirow[t]{2}{*}{$<80$ years } & $90+$ years & 0.001 & -0.067 & 0.069 & 0.974 \\
\hline & & $80-89$ years & 0.010 & -0.045 & 0.064 & 0.728 \\
\hline \multicolumn{7}{|l|}{ Caregiver characteristics } \\
\hline Sex & Female & Male & -0.041 & -0.085 & 0.002 & 0.064 \\
\hline \multirow[t]{5}{*}{ Age $\mathrm{X}$ employment status } & \multirow{2}{*}{$\begin{array}{l}18-49 \text { years and } \\
\text { unemployed }\end{array}$} & $65+$ years and unemployed & 0.026 & -0.096 & 0.149 & 0.677 \\
\hline & & 50-64 years and unemployed & $-\overline{0}(106$ & -0.202 & -0.009 & 0.032 \\
\hline & $\begin{array}{l}\text { Unemployed and } \\
18-49 \text { years }\end{array}$ & Employed and $18-49$ years & $\overline{-} .076$ & -0.167 & 0.014 & 0.098 \\
\hline & $\begin{array}{l}65+\text { vs. } 18-49 \text { years } \\
\text { and unemployed }\end{array}$ & $65+$ vs. $18-49$ years and employed & 0.026 & -0.139 & 0.19 & 0.758 \\
\hline & $\begin{array}{l}50-64 \text { vs. } 18-49 \text { years } \\
\text { and unemployed }\end{array}$ & $50-64$ vs. $18-49$ years and employed & 0.144 & 0.038 & 0.249 & 0.008 \\
\hline \multicolumn{3}{|c|}{$\%$ average daily care for which caregiver is responsible } & 0.000 & 0.000 & 0.001 & 0.378 \\
\hline \multicolumn{3}{|c|}{$\%$ average daily care from visiting nurse/home-helper } & -0.002 & -0.003 & 0.000 & 0.008 \\
\hline \multicolumn{3}{|c|}{ Total average hours of care required per day } & -0.042 & -0.061 & -0.024 & $\begin{array}{l}< \\
0.001\end{array}$ \\
\hline \multirow{4}{*}{$\begin{array}{l}\text { Years providing care for } A D \text { dementia } \\
\text { patient }\end{array}$} & \multirow[t]{4}{*}{$<2$ years } & 2 to $<4$ years & 0.022 & -0.061 & 0.105 & 0.603 \\
\hline & & 4 to $<6$ years & 0.050 & -0.037 & 0.137 & 0.258 \\
\hline & & $6+$ years & 0.025 & -0.062 & 0.112 & 0.575 \\
\hline & & Can't recall & 0.084 & -0.018 & 0.186 & 0.108 \\
\hline \multirow[t]{2}{*}{ Marital status } & \multirow[t]{2}{*}{ Single } & Married / living with partner & 0.011 & -0.037 & 0.059 & 0.660 \\
\hline & & Divorced / separated / widowed & -0.068 & -0.145 & 0.01 & 0.087 \\
\hline \multirow[t]{2}{*}{ Relationship with patient } & \multirow{2}{*}{$\begin{array}{l}\text { In-law, extended, not } \\
\text { related }\end{array}$} & Offspring of patient (caregiver $\leq 20$ years) & 0.021 & -0.031 & 0.074 & 0.420 \\
\hline & & $\begin{array}{l}\text { Spouse / sibling / parent of patient (caregiver > } \\
20 \text { years) }\end{array}$ & -0.048 & -0.166 & 0.07 & 0.424 \\
\hline \multicolumn{3}{|l|}{ (Scale) } & 0.028 & 0.024 & 0.033 & \\
\hline
\end{tabular}

Note. Presented are normal generalized linear model results. $A D$ Alzheimer's disease, $B$ unstandardized beta, $L C L$ lower confidence limit, $U C L$ upper confidence limit 


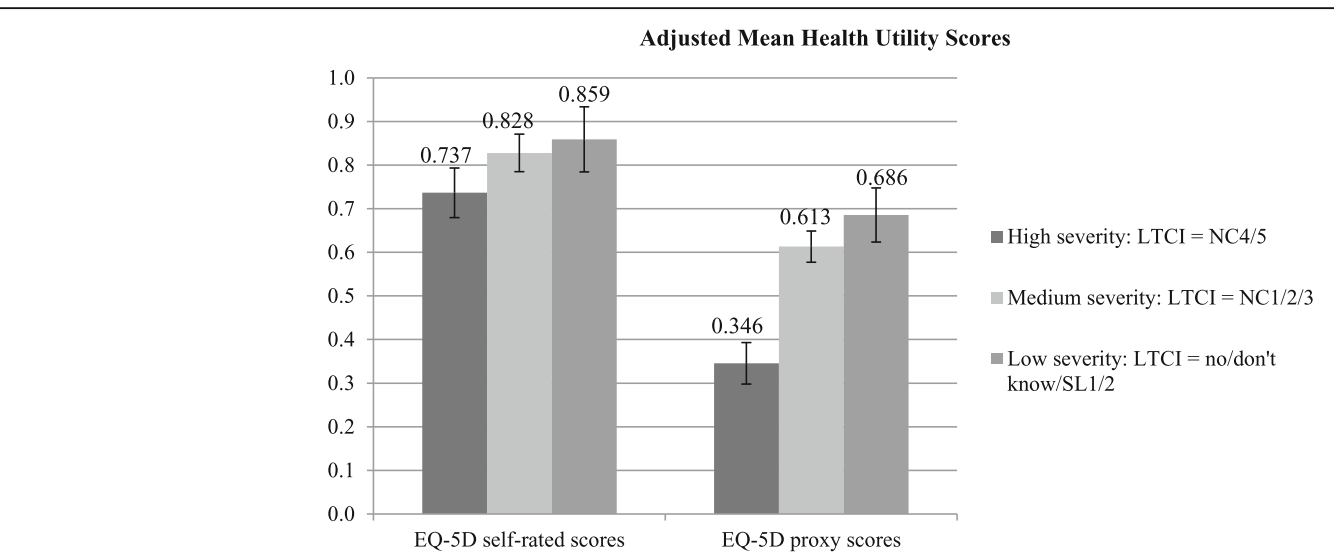

Fig. 2 Adjusted means for health utility scores among AD dementia caregivers and patients. Note. EQ-5D scores are for AD dementia caregivers, EQ-5D proxy scores are for AD dementia patients as reported by their caregivers. AD Alzheimer's disease, LTCI long-term care insurance, NC nursing care level, SL support level

the EQ-5D patient and caregiver models, Age 50-64 X Employed interaction terms were statistically significant (see Table 4 and Table 5). These interaction terms indicate that although employment tended to be associated with poorer HRQoL for caregivers and patients, this difference was reduced among caregivers who were 5064 years-old as well as patients with caregivers who were 50-64 years-old.

Among caregivers, AD dementia severity was not associated with developing any of the measured comorbidities since becoming caregivers, including anxiety, hypertension, and insomnia. AD dementia severity was also not associated with depression severity interval scores or MDD among caregivers as measured by the PHQ-9. PHQ-9 scores indicative of MDD among caregivers were, however, relatively high across $\mathrm{AD}$ dementia severity, including $35.1 \%$ of caregivers of low severity patients, $27.2 \%$ of caregivers of medium severity patients, and $34.7 \%$ of caregivers of high severity patients. Therefore, comorbidities were not explored further as outcomes in multivariable analysis.

\section{Discussion}

The current study quantified the incremental burden associated with greater patient severity and its relationship with poorer patient and caregiver HRQoL. These findings reinforce the vulnerable nature of these two populations, both patients and caregivers, and the need for new interventions that can slow or halt disease progression and maintain or improve patient outcomes. These findings also emphasize the need for comprehensive supportive services for both patients and caregivers alike.

In previous studies, caregivers reported significant and broad impairment when compared with non-caregivers, even in the early stages of disease related to AD dementia or other types of dementia [32]. The current study extends these findings and suggests that severity specific to $\mathrm{AD}$ dementia is associated with greater burden among caregivers, encompassing impairments in both patients' and caregivers' HRQoL. Further, these results provide important and unique insights into the experience of patients and caregivers in the context of mild disease severity.

The current study is also consistent with past research that established that disease severity was associated with poorer patient and caregiver HRQoL $[4,5]$. For example, Hessman and colleagues reported that increasing disease severity, as measured by the Mini-Mental State Examination (MMSE), was associated with significantly worse quality of life among patients in both institutional and community settings [5]. Studies have also suggested that caregivers' quality of life can worsen over time [54], and a study by Kamiya and colleagues found that caring for patients with behavioral disturbances and lower scores on the MMSE (i.e., higher disease severity) was associated with increased caregiver burden [55]. A further study conducted in South Korea reported that more severe disease and greater functional impairment were also associated with increased caregiver burden [56], while a study by Ferrara and colleagues of 200 caregivers reported a proportional relationship between increased disease severity (as measured by MMSE) and caregiver burden (anxiety/ depression). The authors suggested that disease severity was a primary force in the reorganization of the family unit as the level of care needed by the patient increased [57].

The current results, along with previous findings, reinforce the considerable burden associated with $\mathrm{AD}$ dementia, its progressive nature, and the increasing burden that can be associated with caregiving. This line of research emphasizes the need for programs or interventions (e.g., formal assistance programs) to be developed to support caregiving responsibilities and ultimately reduce 
Table 5 Results of generalized linear regression model of caregiver EQ-5D self-rated index values as a function of LTCI \& covariates $(n=300)$

\begin{tabular}{|c|c|c|c|c|c|c|}
\hline Characteristic & Reference Category & Variables & $\mathrm{B}$ & $95 \% \mathrm{LCL}$ & $95 \%$ UCL & $P$-Value \\
\hline (Intercept) & & & 0.913 & 0.754 & 1.073 & $<0.001$ \\
\hline \multicolumn{7}{|l|}{ Patient characteristics } \\
\hline \multirow[t]{2}{*}{ AD dementia severity } & \multirow[t]{2}{*}{ Low severity } & High severity & -0.122 & -0.211 & -0.034 & 0.007 \\
\hline & & Medium severity & -0.031 & -0.105 & 0.043 & 0.414 \\
\hline Sex & Female & Male & 0.003 & -0.055 & 0.061 & 0.916 \\
\hline \multirow[t]{2}{*}{ Age } & \multirow[t]{2}{*}{$<80$ years } & $90+$ years & 0.085 & 0.002 & 0.167 & 0.044 \\
\hline & & $80-89$ years & 0.019 & -0.046 & 0.085 & 0.564 \\
\hline \multicolumn{7}{|l|}{ Caregiver characteristics } \\
\hline Sex & Female & Male & -0.016 & -0.069 & 0.037 & 0.560 \\
\hline \multirow[t]{5}{*}{ Age $\mathrm{X}$ employment status } & \multirow[t]{2}{*}{ 18-49 years and unemployed } & $65+$ years and unemployed & -0.014 & -0.162 & 0.133 & 0.847 \\
\hline & & 50-64 years and unemployed & -0.081 & -0.198 & 0.035 & 0.169 \\
\hline & $\begin{array}{l}\text { Unemployed and 18-49 } \\
\text { years }\end{array}$ & Employed and $18-49$ years & -0.109 & -0.217 & 0.000 & 0.050 \\
\hline & $\begin{array}{l}65+\text { vs. } 18-49 \text { years and } \\
\text { unemployed }\end{array}$ & $\begin{array}{l}65+\text { vs. } 18-49 \text { years and } \\
\text { employed }\end{array}$ & 0.016 & -0.182 & 0.214 & 0.871 \\
\hline & $\begin{array}{l}50-64 \text { vs. } 18-49 \text { years and } \\
\text { unemployed }\end{array}$ & $\begin{array}{l}50-64 \text { vs. } 18-49 \text { years and } \\
\text { employed }\end{array}$ & 0.155 & 0.028 & 0.282 & 0.017 \\
\hline \multicolumn{3}{|c|}{$\%$ average daily care for which caregiver is responsible } & -0.001 & -0.002 & 0.00 & 0.070 \\
\hline \multicolumn{3}{|c|}{$\%$ average daily care from visiting nurse/home-helper } & -0.002 & -0.004 & -0.001 & 0.003 \\
\hline \multicolumn{3}{|c|}{ Total average hours of care required per day } & -0.009 & -0.032 & 0.013 & 0.424 \\
\hline \multirow{4}{*}{$\begin{array}{l}\text { Years providing care for AD dementia } \\
\text { patient }\end{array}$} & \multirow[t]{4}{*}{$<2$ years } & 2 to $<4$ years & 0.001 & -0.098 & 0.100 & 0.984 \\
\hline & & 4 to $<6$ years & 0.030 & -0.075 & 0.135 & 0.573 \\
\hline & & $6+$ years & 0.025 & -0.079 & 0.130 & 0.634 \\
\hline & & Can't recall & 0.002 & -0.121 & 0.125 & 0.972 \\
\hline \multirow[t]{2}{*}{ Marital status } & \multirow[t]{2}{*}{ Single } & Married/living with partner & 0.018 & -0.040 & 0.076 & 0.535 \\
\hline & & Divorced/separated/widowed & -0.021 & -0.114 & 0.073 & 0.663 \\
\hline \multirow[t]{2}{*}{ Relationship with patient } & \multirow[t]{2}{*}{ In-law, extended, not related } & $\begin{array}{l}\text { Offspring of patient (caregiver } \\
\leq 20 \text { years) }\end{array}$ & 0.026 & -0.036 & 0.089 & 0.411 \\
\hline & & $\begin{array}{l}\text { Spouse/sibling/parent of } \\
\text { patient (caregiver }>20 \text { years) }\end{array}$ & -0.022 & -0.164 & 0.120 & 0.762 \\
\hline \multicolumn{3}{|l|}{ (Scale) } & 0.041 & 0.035 & 0.048 & \\
\hline
\end{tabular}

Note. Presented are normal generalized linear model results. AD Alzheimer's disease, $B$ unstandardized beta, $L C L$ lower confidence limit, UCL upper confidence limit

burden and improve outcomes among patients and caregivers alike. For example, there is a cluster-randomized study that assessed psychosocial behavior management programme for home-dwelling people living with dementia [58]. This programme reduced challenging behaviors, which were evaluated by the Neuropsychiatric Inventory. This kind of intervention may also provide a host of benefits for patients, caregivers, and society. The potential to delay disease progression through early detection and prompt initiation of effective treatments/interventions have positive implications for patients' and caregivers' quality of life. Further research is needed to identify pharmacotherapies that can provide sustained relief from symptomatology and ideally modify the trajectory of the disease. Research on other types of interventions to help caregivers throughout the disease progression is also of high importance.

Importantly, a higher proportion of caregivers of $\mathrm{AD}$ dementia patients in this study reported depressive symptomology suggestive of MDD, compared with previous research that included caregivers of patients with dementia defined more broadly [32]. A total of $30.0 \%$ of the caregivers in this study reported PHQ- 9 scores indicative of MDD, compared with $14.2 \%$ of caregivers in the previous study. These findings suggest that caring for AD dementia patients may be associated with greater burden relative to caring for patients with other types of dementia. Contrary to expectation, however, comorbid conditions such as MDD were not associated with severity of $\mathrm{AD}$ dementia, with high rates of depression 
reported among caregivers of those with both high and low severity disease. This suggests that caregivers may suffer from psychological distress even at the early stages of $\mathrm{AD}$ dementia, possibly due to the progressive nature of the disease and consideration of future care needs, and thus reinforcing the need for supportive care and treatment advances. Future studies should examine the relative burden of caregiving on HRQoL based on the type of dementia experienced by patients.

Although significant interactions were found between age (namely, 50-64-year-olds vs. 18-49-year-olds) and employment status, they did not reveal a consistent pattern across age groups. Speculatively, there was a non-significant trend toward poorer HRQoL with employment, suggesting that younger caregivers who were also employed (and potentially caring for children as well) may have tended to have an especially difficult time coping with the multiple demands of caregiving and maintaining a job, which may also have had an effect on patients' HRQoL. This effect was reduced among 50-64-year-olds, perhaps implying that those with more established positions at work or without dependent children in the household were in relatively better shape to cope with the multiple demands.

\section{Study limitations}

The online survey format of the current study provides certain sampling advantages but may under-represent caregivers without access to or sufficient comfort with online technology. This may result in a biased population from which surveys are drawn, such as respondents from higher socioeconomic backgrounds as well as respondents who are relatively young; however, it is important to note that if caregivers with access to and comfort with technology report burden associated with caregiving, the current study results may actually be an underestimation of burden among AD dementia caregivers in Japan.

There were a number of differences in demographic characteristics between the current study sample and the general population in Japan, with respondents in the current study being more likely to be younger in age $(10.7 \%$ vs. $26.7 \%$ over age 65$)$, male ( $55 \%$ vs. $48.7 \%)$, and socioeconomically advantaged (69\% vs. $58.6 \%$ employed) [59]. These differences may reflect greater motivation, physical ability, and Internet access among an online-survey based study sample. Particularly of note is the high proportion of male caregivers, especially relative to the percentage of female patients (78.7\%). Whereas the relatively large representation of male caregivers in this study contrasts with other similar international studies of AD dementia caregivers, in which female caregivers were the majority [60], this study may provide unique insight into a group that is typically underrepresented in this field of study.
A further notable limitation of the current study is the cross-sectional, contemporaneous nature of the data, which precludes causal assessment (e.g., the change in burden with transition across patient disease states within caregivers). For example, greater caregiver burden may lead to the implementation of increasing levels of nursing care, thus resulting in a positive correlation between these two factors. Further research is needed to examine these relationships over time and to best guide future policy and supportive care initiatives.

The current study also utilized a proxy measure of patient HRQoL completed by their caregivers. Whereas evidence suggests this to be appropriate [61], previous studies have shown that discrepancies can exist in the ratings of patient outcomes based on the rater (patient versus caregiver). Whereas spouses or partners who live with the patient have been found to provide the most reliable informant ratings [62], a Taiwan-based study reported that such discrepancies could be associated with the quality of the patient-caregiver relationship, with greater discrepancies reported between dyads with poorer relationships on an HRQoL measure [63]. In a two-year study of $574 \mathrm{AD}$ dementia patients and their caregivers, researchers found that while patient-reported HRQoL was stable over the course of the study, disease severity markers and caregiver-rated QoL declined [64]. Given these possible influencing factors, caution is warranted in interpreting proxy-based assessment of patient outcomes.

\section{Conclusions}

The current findings provide specific quantification of burden among $\mathrm{AD}$ dementia patients and caregivers alike, and suggest that greater AD dementia severity is associated with a higher degree of burden, most notably related to HRQoL. This study also provides important and novel insight into the experience of those with mild disease symptoms, a group that has received little attention in previous research. In the context of an aging global population and the progressive nature of $\mathrm{AD}$ dementia, these results further highlight the need for efforts in promoting early detection and treatment, an issue that has received increasing attention through national organizations in Japan and globally $[65,66]$. New therapies that promise to slow or halt $\mathrm{AD}$ dementia progression (once it has been diagnosed), and thus maintain patients in a milder disease state, are needed. The benefits of early detection and treatment could provide a significant reduction in disease-related burden for patients, caregivers, and society at large.

\section{Abbreviations}

AD: Alzheimer's disease; CCl: Charlson comorbidity index; EQ-5D: EuroQol5Dimension; GCDI: Government-certified Disability Index; HRQoL: Healthrelated quality of life; LTCl: Long-term nursing care insurance; MDD: Major 
depressive disorder; MMSE: Mini-Mental State Examination; NHWS: National Health and Wellness Survey; PHQ: Patient Health Questionnaire; SD: Standard deviation; SMQ: Short-Memory Questionnaire

\section{Acknowledgments}

The authors acknowledge Michael J. Doane, PhD and Colleen M. Carpinella, PhD (at Kantar Health) and Errol J. Philip, PhD for assistance with literature review and writing.

\section{Funding}

This study, including study design, data collection, analysis, interpretation, and reporting, was funded by Eli Lilly and Company, as was the development of this manuscript and publication fees.

\section{Availability of data and materials}

Survey data used in this study will be made available for non-commercial research and validation purposes, upon request.

\section{Authors' contributions}

WM, KKW, TN, KU, and AG contributed to study design, interpretation, and writing/review of this publication, including approval of the final manuscript. AG performed the statistical analyses. AG and Kantar Health conducted the data collection.

\section{Ethics approval and consent to participate}

The original NHWS patient protocol and questionnaire were reviewed and approved by Essex Institutional Review Board (Lebanon, New Jersey, USA). The follow-up survey was reviewed and approved by Pearl Institutional Review Board (Indianapolis, Indiana, USA). All participants provided their explicit informed consent online, given that this was an internet-based questionnaire.

\section{Competing interests}

William Montgomery, Kristin Kahle-Wrobleski, Tomomi Nakamura, and Kaname Ueda were full-time employees of Eli Lilly and Company at the time of the study. Amir Goren is an employee of Kantar Health, which conducted this study with funding from Eli Lilly, and manages the National Health and Wellness Survey.

\section{Publisher's Note}

Springer Nature remains neutral with regard to jurisdictional claims in published maps and institutional affiliations.

\section{Author details}

'Global Patient Outcomes \& Real World Evidence, Eli Lilly Australia, 112 Wharf Rd, West Ryde, NSW 2114, Australia. ${ }^{2}$ Health Outcomes Practice, Kantar Health, 11 Madison Ave, Floor 12, New York, NY 10010, USA. ${ }^{3}$ Global Patient Outcomes \& Real World Evidence, Eli Lilly and Company, Lilly Corporate Center, Indianapolis, IN 46285, USA. ${ }^{4}$ Medical Development Unit, Eli Lilly Japan K.K, 4-15-1, Akasaka, Minato-ku, Tokyo 107-0052, Japan. ${ }^{5}$ Health Outcomes, Health Technology Assessment, \& Real World Evidence, Medical Development Unit, Eli Lilly Japan K.K, 5-1-28, Isogami-dori, chuou-ku, Kobe 651-0086, Japan.

Received: 10 January 2018 Accepted: 29 May 2018

Published online: 14 June 2018

\section{References}

1. National Institute on Aging. Alzheimer's Disease Fact Sheet 2015 [Available from: http://www.nia.nih.gov/alzheimers/publication/ alzheimers-disease-fact-sheet.

2. Alzheimer's Association. 2014 Alzheimer's disease facts and figures. Alzheimers Dement. 2014;10:e47-92.

3. Brodaty $H$, Donkin M. Family caregivers of people with dementia. Dialogues Clin Neurosci. 2009;11:217-28.

4. Conde-Sala JL, Turro-Garriga O, Pinan-Hernandez S, Portellano-Ortiz C, VinasDiez V, Gascon-Bayarri J, et al. Effects of anosognosia and neuropsychiatric symptoms on the quality of life of patients with Alzheimer's disease: a 24month follow-up study. Int J Geriatr Psychiatry. 2016;31:109-19.
5. Hessmann P, Seeberg G, Reese JP, Dams J, Baum E, Muller MJ, et al. Healthrelated quality of life in patients with Alzheimer's disease in different German health care settings. J Alzheimers Dis. 2016;51:545-61.

6. Wimo A, Reed CC, Dodel R, Belger M, Jones RW, Happich M, et al. The GERAS study: a prospective observational study of costs and resource use in community dwellers with Alzheimer's disease in three European countriesstudy design and baseline findings. J Alzheimers Dis. 2013;36:385-99.

7. Wolstenholme J, Fenn P, Gray A, Keene J, Jacoby R, Hope T. Estimating the relationship between disease progression and cost of care in dementia. $\mathrm{Br}$ J Psychiatry. 2002;181:36-42

8. Jonsson L, Eriksdotter Jonhagen M, Kilander L, Soininen $H$, Hallikainen M, Waldemar G, et al. Determinants of costs of care for patients with Alzheimer's disease. Int J Geriatr Psychiatry. 2006;21:449-59.

9. Handels RL, Wolfs CA, Aalten P, Verhey FR, Severens JL. Determinants of care costs of patients with dementia or cognitive impairment. Alzheimer Dis Assoc Disord. 2013;27:30-6

10. Small GW, McDonnell DD, Brooks RL, Papadopoulos G. The impact of symptom severity on the cost of Alzheimer's disease. J Am Geriatr Soc. 2002;50:321-7.

11. Souetre EJ, Qing W, Vigoureux I, Dartigues JF, Lozet H, Lacomblez L, et al. Economic analysis of Alzheimer's disease in outpatients: impact of symptom severity. Int Psychogeriatr. 1995;7:115-22.

12. Quentin W, Riedel-Heller SG, Luppa M, Rudolph A, Konig HH. Cost-of-illness studies of dementia: a systematic review focusing on stage dependency of costs. Acta Psychiatr Scand. 2010;121:243-59.

13. Moore MJ, Zhu CW, Clipp EC. Informal costs of dementia care: estimates from the National Longitudinal Caregiver Study. J Gerontol B Psychol Sci Soc Sci. 2001:56:S219-28.

14. Hearson B, McClement S. Sleep disturbance in family caregivers of patients with advanced cancer. Int J Palliat Nurs. 2007:13:495-501.

15. Schulz R, Beach SR. Caregiving as a risk factor for mortality: the caregiver health effects study. JAMA. 1999:282:2215-9.

16. Schulz R, O'Brien AT, Bookwala J, Fleissner K. Psychiatric and physical morbidity effects of dementia caregiving: prevalence, correlates, and causes. Gerontologist. 1995;35:771-91.

17. Bergvall N, Brinck P, Eek D, Gustavsson A, Wimo A, Winblad B, et al. Relative importance of patient disease indicators on informal care and caregiver burden in Alzheimer's disease. Int Psychogeriatr. 2011:23:73-85.

18. Laks J, Goren A, Duenas H, Novick D, Kahle-Wrobleski K. Caregiving for patients with Alzheimer's disease or dementia and its association with psychiatric and clinical comorbidities and other health outcomes in Brazil. Int J Geriatr Psychiatry. 2015

19. Fuller-Jonap F, Haley WE. Mental and physical health of male caregivers of a spouse with Alzheimer's disease. J Aging Health. 1995;7:99-118.

20. Kolanowski AM, Fick D, Waller UL, Shea D. Spouses of persons with dementia: their healthcare problems, utilization, and costs. Res Nurs Health. 2004:27:296-306.

21. Lu YF, Wykle M. Relationships between caregiver stress and self-care behaviors in response to symptoms. Clin Nurs Res. 2007:16:29-43.

22. Beeson R, Horton-Deutsch S, Farran C, Neundorfer M. Loneliness and depression in caregivers of persons with Alzheimer's disease or related disorders. Issues Ment Health Nurs. 2000;21:779-806.

23. Langa KM, Chernew ME, Kabeto MU, Herzog AR, Ofstedal MB, Willis RJ, et al. National estimates of the quantity and cost of informal caregiving for the elderly with dementia. J Gen Intern Med. 2001;16:770-8.

24. Arai A, Matsumoto T, Ikeda M, Arai Y. Do family caregivers perceive more difficulty when they look after patients with early onset dementia compared to those with late onset dementia? Int J Geriatr Psychiatry. 2007;22:1255-61.

25. Arai Y. [Medical and nursing care of dementia patients. Nursing burden of family caregivers] Nihon Naika Gakkai Zasshi 2005;94:1548-54.

26. Arai $Y$, Kumamoto $K$, Washio M, Ueda T, Miura H, Kudo K. Factors related to feelings of burden among caregivers looking after impaired elderly in Japan under the long-term care insurance system. Psychiatry Clin Neurosci. 2004; 58:396-402

27. Arai Y, Zarit SH. Exploring strategies to alleviate caregiver burden: effects of the National Long-Term Care insurance scheme in Japan. Psychogeriatrics. 2011;11:183-9.

28. Kurasawa S, Yoshimasu K, Washio M, Fukumoto J, Takemura S, Yokoi K, et al. Factors influencing caregivers' burden among family caregivers and institutionalization of in-home elderly people cared for by family caregivers. Environ Health Prev Med. 2012;17:474-83. 
29. Sasaki M, Arai Y, Kumamoto K, Abe K, Arai A, Mizuno Y. Factors related to potentially harmful behaviors towards disabled older people by family caregivers in Japan. Int J Geriatr Psychiatry. 2007;22:250-7.

30. Hayashi S, Terada S, Nagao S, Ikeda C, Shindo A, Oshima E, et al. Burden of caregivers for patients with mild cognitive impairment in Japan. Int Psychogeriatr. 2013;25:1357-63.

31. Muraki I, Yamagishi K, Ito Y, Fujieda T, Ishikawa Y, Miyagawa Y, et al. Caregiver burden for impaired elderly Japanese with prevalent stroke and dementia under long-term care insurance system. Cerebrovasc Dis. 2008;25:234-40.

32. Goren A, Montgomery W, Kahle-Wrobleski K, Nakamura T, Ueda K. Impact of caring for persons with Alzheimer's disease or dementia on caregivers' health outcomes: findings from a community based survey in Japan. BMC Geriatr. 2016;16:122.

33. Cassidy T. Benefit finding through caring: the cancer caregiver experience. Psychol Health. 2013;28:250-66.

34. Cheng ST, Lau RW, Mak EP, Ng NS, Lam LC. Benefit-finding intervention for Alzheimer caregivers: conceptual framework, implementation issues, and preliminary efficacy. Gerontologist. 2014;54:1049-58.

35. Kim Y, Schulz R, Carver CS. Benefit-finding in the cancer caregiving experience. Psychosom Med. 2007;69:283-91.

36. Lum HD, Lo D, Hooker S, Bekelman DB. Caregiving in heart failure: relationship quality is associated with caregiver benefit finding and caregiver burden. Heart Lung. 2014:43:306-10.

37. Duthey B. Background paper 6.11: Alzheimer disease and other dementias: A Public Health Approach to Innovation (Update on 2004 Background Paper) pp 1-74; 2013 [Available from: http://www.who.int/medicines/areas/ priority_medicines/BP6_11Alzheimer.pdf.

38. Ninomiya et al. Report of "A study on the future estimates of the elderly population of dementia in Japan" (direct translation, not official). 2015 March.

39. Sugino H, Watanabe A, Amada N, Yamamoto M, Ohgi Y, Kostic D, et al. Global trends in Alzheimer disease clinical development: increasing the probability of success. Clin Ther. 2015;37:1632-42.

40. Laks J, Goren A, Duenas H, Novick D, Kahle-Wrobleski K. Caregiving for patients with Alzheimer's disease or dementia and its association with psychiatric and clinical comorbidities and other health outcomes in Brazil. Int J Geriatr Psychiatry. 2016;31:176-85.

41. Arai $Y$, Zarit S, Kumamoto K, Takeda A. Are there inequities in the assessment of dementia under Japan's LTC insurance system? Int J Geriatr Psychiatry. 2003;18:346-52.

42. Montgomery W, Goren A, Kahle-Wrobleski K, Nakamura T, Ueda K. Detection, diagnosis, and treatment of Alzheimer's disease dementia stratified by severity as reported by caregivers in Japan. Neuropsychiatr Dis Treat. 2018;14:1-12.

43. Koss E, Patterson MB, Ownby R, Stuckey JC, Whitehouse PJ. Memory evaluation in Alzheimer's disease. Caregivers' appraisals and objective testing. Arch Neurol. 1993;50:92-7.

44. Maki N, Ikeda M, Hokoishi K, Nebu A, Komori K, Hirono N, et al. Japanese version of the short-memory questionnaire: memory evaluation in Alzheimer's disease. No To Shinkei. 1998;50:415-8.

45. Charlson ME, Pompei $P$, Ales KL, MacKenzie CR. A new method of classifying prognostic comorbidity in longitudinal studies: development and validation. J Chronic Dis. 1987;40:373-83.

46. Quan H, Li B, Couris CM, Fushimi K, Graham P, Hider P, et al. Updating and validating the Charlson comorbidity index and score for risk adjustment in hospital discharge abstracts using data from 6 countries. Am J Epidemiol. 2011;173:676-82

47. EuroQol-a new facility for the measurement of health-related quality of life. Health Policy. 1990;16:199-208.

48. Team JEQT. The development of the Japanese EuroQol instrument. J Health Care Soc. 1998:8:109-23.

49. Dolan P. Modeling valuations for EuroQol health states. Med Care. 1997;35: 1095-108.

50. Tsuchiya A, Ikeda S, Ikegami N, Nishimura S, Sakai I, Fukuda T, et al. Estimating an EQ-5D population value set: the case of Japan. Health Econ. 2002;11:341-53.

51. Kroenke K, Spitzer RL, Williams JB. The PHQ-9: validity of a brief depression severity measure. J Gen Intern Med. 2001;16:606-13.

52. Muramatsu K, Miyaoka H, Kamijima K, Muramatsu Y, Yoshida M, Otsubo T, et al. The patient health questionnaire, Japanese version: validity according to the mini-international neuropsychiatric interview-plus. Psychol Rep. 2007; 101:952-60.

53. Manea L, Gilbody S, McMillan D. Optimal cut-off score for diagnosing depression with the patient health questionnaire (PHQ-9): a meta-analysis. CMAJ. 2012;184:E191-6.

54. Garzon-Maldonado FJ, Gutierrez-Bedmar M, Garcia-Casares N, PerezErrazquin F, Gallardo-Tur A, Martinez-Valle Torres MD. Health-related quality of life in caregivers of patients with Alzheimer's disease. Neurologia. 2016

55. Kamiya M, Sakurai T, Ogama N, Maki Y, Toba K. Factors associated with increased caregivers' burden in several cognitive stages of Alzheimer's disease. Geriatr Gerontol Int. 2014;14(Suppl 2):45-55.

56. Kang HS, Myung W, Na DL, Kim SY, Lee JH, Han SH, et al. Factors associated with caregiver burden in patients with Alzheimer's disease. Psychiatry Investig. 2014;11:152-9.

57. Ferrara M, Langiano E, Di Brango T, De Vito E, Di Cioccio L, Bauco C. Prevalence of stress, anxiety and depression in with Alzheimer caregivers. Health Qual Life Outcomes. 2008;6:93.

58. Nakanishi M, Endo K, Hirooka K, Granvik E, Minthon L, Nagga K, et al. Psychosocial behaviour management programme for home-dwelling people with dementia: a cluster-randomized controlled trial. Int J Geriatr Psychiatry. 2018;33:495-503.

59. Statistics Bureau. Statistics Japan. Ministry of Internal Affairs and Communications; 2016.

60. Laks J, Goren A. Caregiving for patients with Alzheimer's disease or dementia and its association with psychiatric and clinical comorbidities and other health outcomes in Brazil. 2016:31:176-185.

61. Diaz-Redondo A, Rodriguez-Blazquez C, Ayala A, Martinez-Martin P, Forjaz MJ. EQ-5D rated by proxy in institutionalized older adults with dementia: psychometric pros and cons. Geriatr Gerontol Int. 2014;14:346-53.

62. Ready RE, Ott BR, Grace J. Validity of informant reports about $A D$ and $M C$ patients' memory. Alzheimer Dis Assoc Disord. 2004;18:11-6.

63. Huang HL, Chang MY, Tang JS, Chiu YC, Weng LC. Determinants of the discrepancy in patient- and caregiver-rated quality of life for persons with dementia. J Clin Nurs. 2009;18:3107-18.

64. Andrieu S, Coley N, Rolland Y, Cantet C, Arnaud C, Guyonnet S, et al. Assessing Alzheimer's disease patients' quality of life: discrepancies between patient and caregiver perspectives. Alzheimers Dement. 2016;12:427-37.

65. Alzheimer's Association. Early detection and diagnosis of Alzheimer's disease. 2015

66. Alzheimer's Association. Alzheimer's and Dementia in Japan: Alzheimer's Association; 2016 [Available from: http://www.alz.org/jp/dementiaalzheimers-japan.asp.

\section{Ready to submit your research? Choose BMC and benefit from:}

- fast, convenient online submission

- thorough peer review by experienced researchers in your field

- rapid publication on acceptance

- support for research data, including large and complex data types

- gold Open Access which fosters wider collaboration and increased citations

- maximum visibility for your research: over $100 \mathrm{M}$ website views per year

At BMC, research is always in progress.

Learn more biomedcentral.com/submissions 